Advances in Geosciences, 4, 63-68, 2005

SRef-ID: $1680-7359 /$ adgeo/2005-4-63

European Geosciences Union

(c) 2005 Author(s). This work is licensed

under a Creative Commons License.

\title{
Spatial modelling of air pollution in urban areas with GIS: a case study on integrated database development
}

\author{
L. Matejicek \\ Institute for Environmental Studies, Charles University, Prague, 128 01, Czech Republic \\ Received: 1 August 2004 - Revised: 1 November 2004 - Accepted: 15 November 2004 - Published: 9 August 2005
}

\begin{abstract}
A wide range of data collected by monitoring systems and by mathematical and physical modelling can be managed in the frame of spatial models developed in GIS. In addition to data management and standard environmental analysis of air pollution, data from remote sensing (aerial and satellite images) can ehance all data sets. In spite of the fact that simulation of air pollutant distribution is carried out by standalone computer systems, the spatial database in the framework of the GIS is used to support decision-making processes in a more efficient way. Mostly, data are included in the map layers as attributes. Other map layers are carried out by the methods of spatial interpolation, raster algebra, and case oriented analysis. A series of extensions is built into the GIS to adapt its functionality. As examples, the spatial models of a flat urban area and a street canyon with extensive traffic polluted with $\mathrm{NO}_{\mathrm{x}}$ are constructed. Different scales of the spatial models require variable methods of construction, data management, and spatial data sources. The measurement of $\mathrm{NO}_{\mathrm{x}}$ and $\mathrm{O}_{3}$ by an automatic monitoring system and data from the differential absorption LIDAR are used for investigation of air pollution. Spatial data contain digital maps of both areas, complemented by digital elevation models. Environmental analyses represent spatial interpolations of air pollution that are displayed in horizontal and vertical planes. Case oriented analyses are mostly focused on risk assessment methods. Finally, the LIDAR monitoring results and the results obtained by modelling and spatial analyses are discussed in the context of environmental management of the urban areas. The spatial models and their extensions are developed in the framework of the ESRI's ArcGIS and ArcView programming tools. Aerial and satellite images preprocessed by the ERDAS Imagine represent areas of Prague.
\end{abstract}

\section{Introduction}

The recent development of spatial data management in the framework of geographic information systems (GISs) has created a new era of environmental modelling. More powerful computers have made running air quality models at global and local spatial scales possible. In order to understand the function of more complex models, the modelling system should consist of other subsystems (point and area sources of pollution, spatial description of terrain elevations, meteorological data, air quality monitoring networks). Obviously, the use of GIS has become essential in providing boundary conditions to the air quality models. Certainly, the use of GIS in air pollution modelling can be further extended to processing the surface data. Many models have been coupled with GIS in the past decade to simulate various environmental processes as described by Longley et al. (2001). Due to the four-dimensional nature of the distribution of atmospheric pollutants, the concept of GIS should be extended to include temporal variations of three-dimensional spatial data. The interpolations, integrations of land cover surface data, and the GIS analyses focused on small scale spatial models carried out in the kilometer grid are discussed by Lee in the book published by Goodchild (1996) and in the frame of particular studies (Matejicek, 1996, 1998, 1999). In case of large scale air quality modelling, more detailed spatial data are needed to include the impact of buildings and other manmade barriers on the distribution of air pollutants, (Janour, 1999; Civis, 2001). Apart from this approach, the statistical theory is also used to indicate spatio-temporal interactions as described by Briggs et al. (2000). 


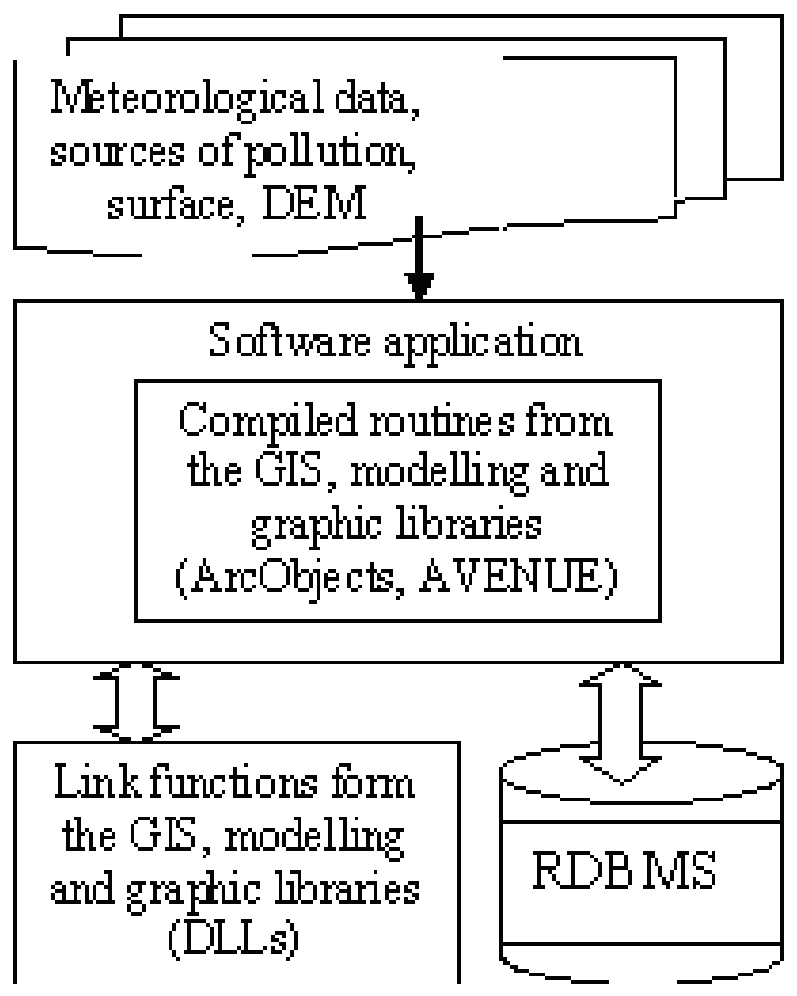

Fig. 1. The standalone software application for integrated evaluation of air quality.

\section{Methods of integration air quality models into GIS}

A few scenarios can be established to integrate air quality models into GIS. The basic level is represented by the standalone software application for simulation of air quality models (ISCST3, ISC-PRIME), which is accompanied by data inputs and outputs. All data can be used independently by other software systems (GIS, RDBMS, Surfer, WWWpresentations). The individual programs form heterogeneous data structures that require the transport of data into various data formats. On the other hand, a number of computer programs have been developed to integrate particular functions of the GIS, air quality modelling, and graphic systems. Mostly, they are designed to carry out specific calculations without links to other software applications. GIS based software applications are mostly based on spatial software libraries. The missing functions (air quality modelling, visualisation tools) can be complemented or shared through dynamic-link libraries. The integrated emission evaluation systems, which offer alternative ways of using the emission models together with selected functionality of GISs, are described by Rebolj (1999). A number of software applications are focused on the design of relational databases and their interconnection together with standard air quality modelling systems. The structure of the programs developed with spatial software libraries is shown in Fig. 1.
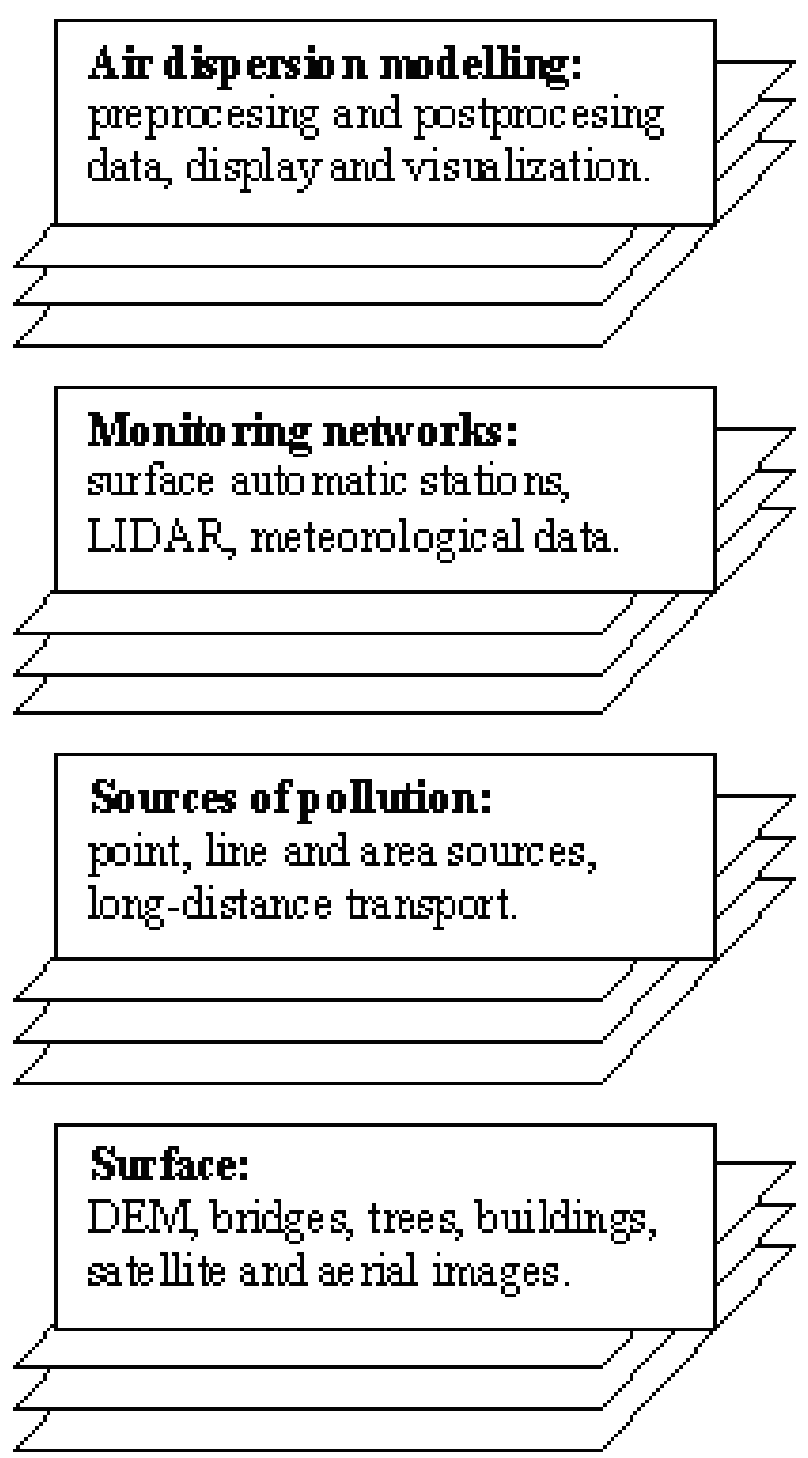

\section{Other data:} general map thernes, data shared from other sources.

Fig. 2. Data included into GIS map layers.

\subsection{GIS data management and functionality}

Considering both described scenarios of integration, the scope and scale of urban area problems make GIS a powerful tool for management of spatial and temporal data, complex analyses, and visualization, (Matejicek, 2002). Due to the ability to manage a number of spatial and temporal data formats, data structures created in the framework of the GISs open the way to building air quality information systems that synthesize geospatial and temporal air quality data to support spatio-temporal analysis and dynamic modelling. There 
is also a growing amount of digital maps in the GIS community, which are used to support decision-making processes of urban authorities (data sets for land cover and climatic variables, digital elevation models, which are extended by blocks of buildings and trees, air pollution sources and monitoring networks, soil and hydrologic properties, road and railway networks). While much progress has been made with the mapping of environmental data and the creation of national, regional, and local data sets, many challenges remain. For example, air quality models are not regularly included into GIS. As standalone software applications, they use various data formats, which can usually operate independently with their own GIS database. Similarly, air quality management agencies are creating GIS data sets to support their operations, without any data standards that can support spatiotemporal analysis and dynamic modelling. The common theme among these challenges is the need for the integrations of different spatial and air quality data, integration of data and modelling, and integration across spatial scales. The requirements for the integrated spatial modelling of air quality in the framework of GIS represent a common geospatial coordinate system, vector themes (points, lines and areas) for description of surface objects (buildings, bridges, vegetation) supported by raster and TIN surface data (digital elevation models), and vector themes for representation of air pollution inputs (local point, line and area sources of pollution, long-distance transport of air pollution). The key parts of the projects represent data of air quality measured by monitoring networks, terrain measurements (LIDAR) and simulation results of air quality models.

\subsection{GIS data models}

At present, all the mentioned properties can be accomplished by few of the GISs. In the presented study, the ArcGIS, distributed by the Environmental Systems Research Institute (ESRI), has been used for the proposed operations. The ArcGIS, a descendant of the widely used ArcInfo, can manage spatial data in various levels, such as shapefiles, coverages, and geodatabases. Moreover, the ArcGIS functionality is expanded by the COM technology, which uses Visual Basic as the standard interface language, just as Microsoft uses the Visual Basic as the interface language for other software applications. The ArcGIS can be customized for particular applications of GIS using specially designed data models. Currently, a number of data models have been published in hydrology (Maidment, 2002), biodiversity, forestry, etc. Air quality modelling can be accomplished by exchanging data between ArcGIS and the independent air quality simulation system, by constructing simulation tools attached to a project in the ArcGIS, or by customizing the behavior of the ArcGIS objects. The choice depends on the model complexity and calculation requirements in the framework of the various ArcGIS levels. All data are stored in the relational database, which can be represented on the basic level by the personal geodatabase (Microsoft Access), or by the RDBMS (Oracle, Microsoft SQL Server). So, the data transfer among

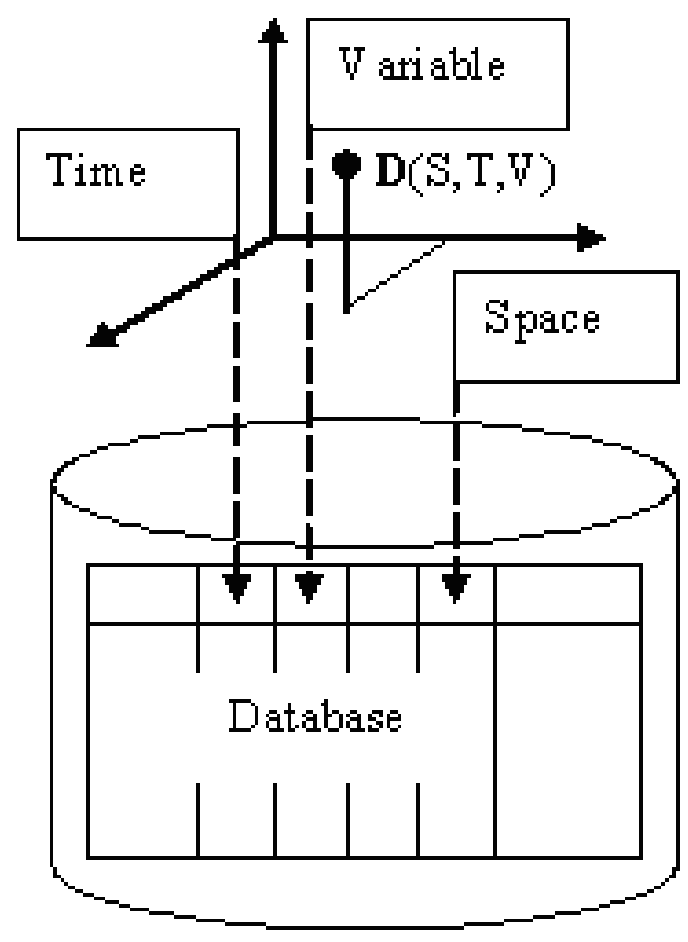

Fig. 3. Data repository with 3-D space indexes.

other standalone software applications can be realized directly through the implemented database connections. In case of the ArcGIS's geodatabase, all the data are loaded into the relational database, so that the geospatial coordinate data of the GIS data layers are stored in the relational data tables. Since the relational database supports relationships between its tables, feature-to-feature spatial connections can be set up among the GIS data layers together with linking and joining of external data tables.

2.3 Spatial models for air quality assessment extended by the LIDAR measurements

The data required for spatial models to serve air quality modelling can be grouped into a few classes. Figure 2 shows spatial data included into map layers in the frame of a GIS project. It is impossible to completely enumerate all the spatial and non-spatial data needed, since the more that is known, the better. However, the accuracy of the model results does not depend on the data alone. The choice of appropriate modelling tools and their settings represents other key parts of air quality modelling. So, if the models do not require or are not capable of evaluating some detailed information, there is little benefit in putting that data in a GIS project. To examine the functionality of the spatial modelling system, the version of the Industrial Source Complex-Short Term (ISCST3) with Plume Rise Enhancements (ISC-PRIME), and the AMS/EPA Regulatory Models (AERMOD/AERMOD-PRIME) have been included into the projects. The ISC-AERMOD View with its preprocessing 


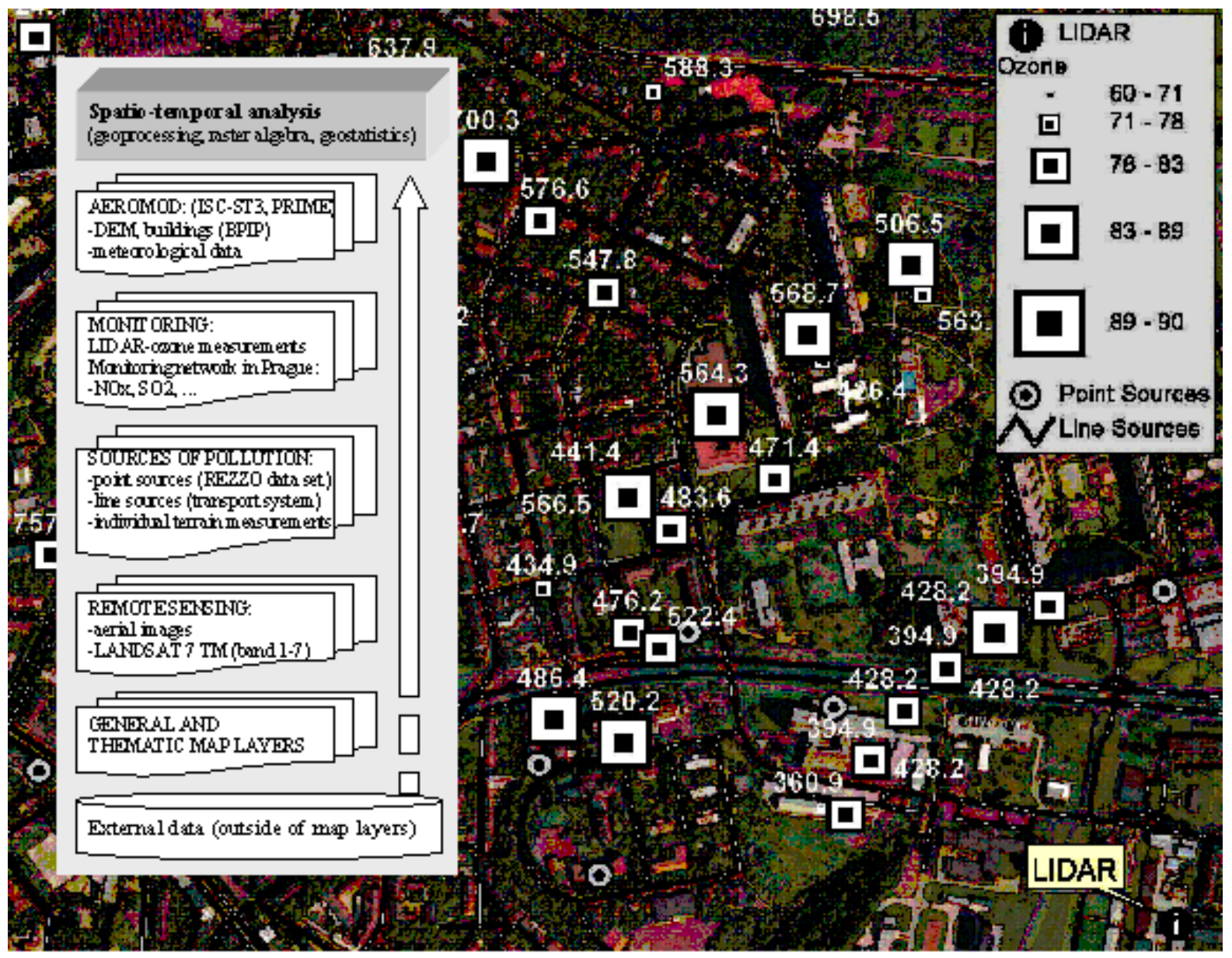

Fig. 4. Map layers of the flat urban area.

and postprocessing modules has been used as the unified interface of the air dispersion models.

The spatial surface data (digital elevation model-DEM, buildings) make up the input into the preprocessing modules (Import of the Digital terrain data in ISC-AERMOD, Building Profile Input Program-BPIP). Other surface data (bridges, trees, satellite and aerial images) complement spatial information for display and visualization. The layers with sources of pollution contain (in addition to the coordinates and shapes) the attributes, which describe emission properties. The surface data and data about sources of pollution have to be transferred into appropriate input formats to run the air quality dispersion models. The primary storage in the GIS spatial database serves furthermore for spatial analysis, display and visualization. As with the previous data, meteorological data are also preprocessed from the database storage into the input formats for air quality dispersion modelling. The map layers, which represent monitoring networks and LIDAR measurements, serve for the comparison of the measured data with the predicted air pollution data calculated by the models.

The mentioned air quality models are steady-state Gaussian plume models used to assess pollutant concentrations from a wide variety of sources mostly associated with an in- dustrial complex. The steady state values of variables are transferred and incorporated into the GIS database, which can be useful in managing data time series. To accommodate large data sets and many variables such as air quality data, climatic data, and properties of sources of pollution, a data repository containing all types of time series data for all features and for all times is proposed.Time series information can thereby be depicted in 3-D space. The three coordinate axes mark space ( $\mathrm{S}$ - identification code of a spatial future), time ( $\mathrm{T}$ - discrete time) and the variable being measured (V - identification code of a variable). The data value indexed by the space, the time and the variable can be defined as $\mathrm{D}(\mathrm{S}, \mathrm{T}, \mathrm{V})$. Thus, each stored value is represented by a point in three-dimensional space with its corresponding coordinates (Fig. 3). In order to extract time series, the space and variable coordinates have to be specified in a query. The result is represented by selected records that match the conditions of the query. Due to spatial properties of the GIS, space coordinates can be derived from a spatial query in the frame of GIS functionality. The associations between the data repository and spatial objects in the ArcGIS geodatabase are specified by relationships, which are stored into the relationship classes. 


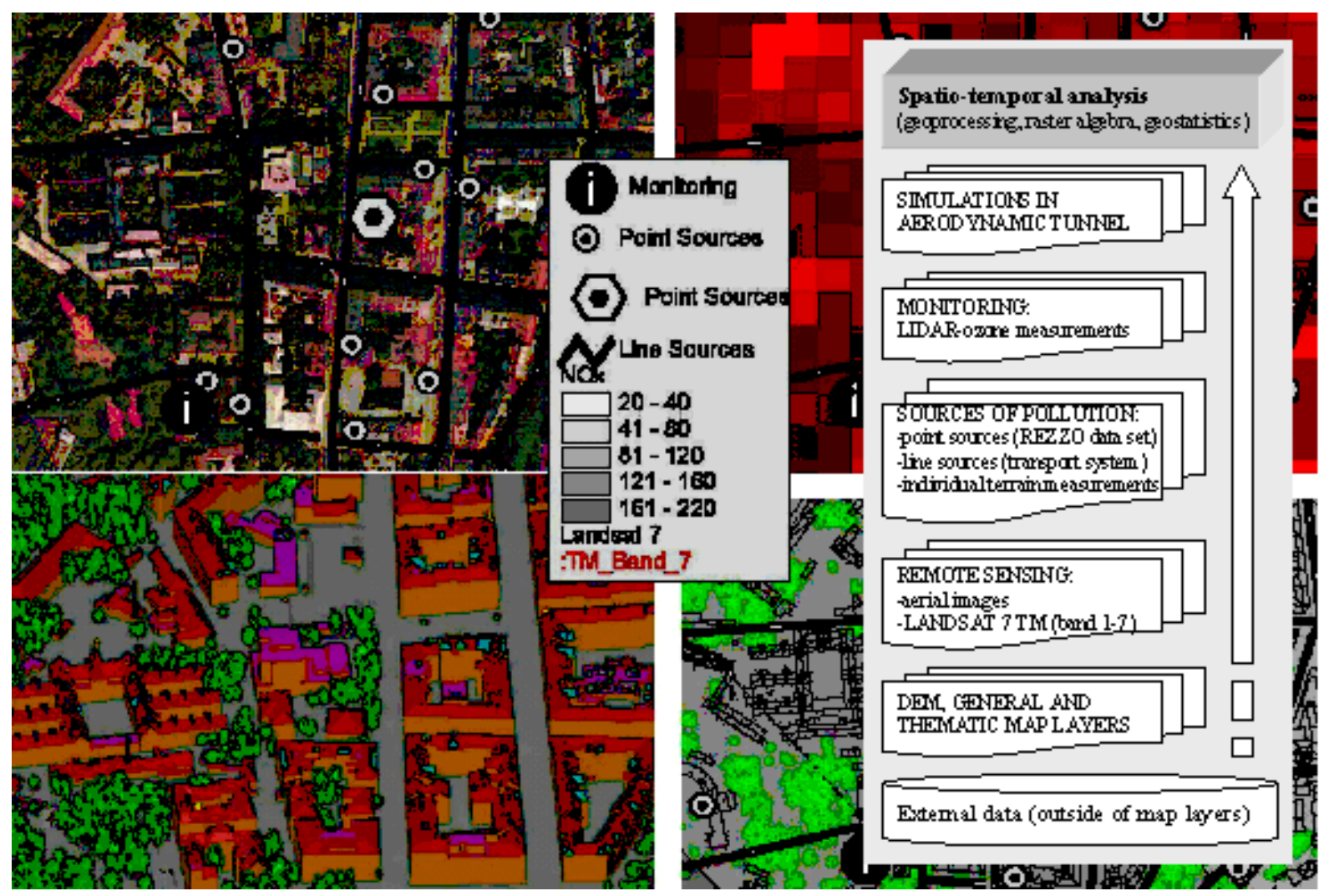

Fig. 5. Map layers of the street canyons.

\section{Case studies of the urban areas}

The various data sets (digital maps, aerial and satellite images, spatio-temporal data in the 3-D database, data outputs from simulation systems) have been linked together to make up projects for different spatial scales. The GIS, originally design to display 2-D digital maps, has been extended into 3-D mapping and data management in the framework of the ArcGIS. As examples, two urban areas of Prague have been used to demonstrate the abilities of spatial modelling.

\subsection{Spatial modelling of a flat urban area}

The inputs of spatial data represent a digital elevation model, which can be used for air pollution modelling, and aerial or satellite images, which can serve for classification of the surface into classes to define the surface graininess and temperature. The sources of air pollution are mapped into a few categories according to the volume of pollution. Their locations and shapes (in case of the line and area sources) together with the attributes are stored in separate themes. Influential sources of pollution, among others, are represented by $\mathrm{NO}_{\mathrm{x}}$ (mostly traffic-related air pollution mapped as the line sources) and $\mathrm{SO}_{2}$ (mostly stationary air pollution registered as the point sources). In addition to data from an automatic monitoring system, the LIDAR (Zelinger, 2003), has been used to complete the data sets. The map composition, which contains the aerial images complemented by the layers with sources of air pollution and 3-D LIDAR data $\left(\mathrm{O}_{3}\right.$ concentration labelled by the elevation), is illustrated in Fig. 4.

\subsection{Spatial modelling of the street canyons}

The streets surrounded by high buildings, in urban areas polluted with traffic-related sources, are spatially modelled as the street canyons. Accumulation of air pollution (mostly from cars) results in high concentrations of organic and inorganic compounds in the street canyons. Distribution and local accumulation of pollutants can be solved by mathematical and physical modelling. In the first stage, the digital terrain model complemented by buildings and other terrain objects is needed to support air quality modelling. Consequently, a complex analysis of all spatio-temporal data has to be performed. Spatial modelling in the framework of the GIS can help to accomplish nearly all these tasks. To demonstrate GIS suitability, a case study of spatial modelling of air quality in urban streets is illustrated in Fig. 5. The map compositions contain various sets of themes. The first part shows the aerial images of the studied local area complemented by the layers with sources of air pollution and one point of the monitoring network. Other map compositions contain the same area complemented by the satellite image from Landsat 7 (the 7th band, which refers to temperature of the surface), the digital terrain model with buildings and trees, and 
a sample of the spatial interpolation of air pollution in the area. Again, in addition to standard analysis, the LIDAR system and the results of physical modelling in the scaled down models (simulations in wind tunnels) can be used to complete the data sets.

\section{Conclusions}

Spatial modelling of air quality in this paper is mainly focused on the integration of a wide range of data in the framework of the GIS spatial database. This method of data management and analysis is also promoted by the LIDAR data, which represent measurements of compounds above the surface located by 3-D coordinates. Despite the complexity of the spatial data management, analysis, and visualization, modelling of air pollution has to be solved independently in the framework of standalone computer systems (mathematical modelling or physical scaled models). The GISs therefore serve as the data stores, which can manage all the data together with model outputs to carry out risk assessment analysis and map compositions. The spatial modelling of street canyons in the framework of the larger urban area complemented by the 3-D LIDAR measurements requires more detailed three dimensional mapping that can generate an extensive volume of data. The spatial modelling of air pollution extended by air dispersion models under a united interface can therefore be used, when supported by adequate hardware, software, and data.

Acknowledgements. The paper was carried out in the frame of the project AVCR 1ET400760405, which is generally focused on measurement and modelling of air pollution in urban areas and flat landscape. The GIS projects were realized in the GIS Laboratory supported by the Ministry of Education, Youth and Sports of the Czech Republic in the frame of the project MSM 113100007 of the Faculty of Natural Science, Charles University in Prague. The digital maps used in the case studies are administrated by the Institute of Municipal Informatics of Prague.

Edited by: P. Krause, S. Kralisch, and W. Flügel

Reviewed by: anonymous referees

\section{References}

Briggs, D. J.: A regression-based method for mapping trafficrelated air pollution: application and testing in four contrasting urban environments, The science of the total environment, 253, 151-167, 2000.

Civis, S., Zelinger, Z., Strizik, M., and Janour, Z.: Simulation of Air Pollution in a Wind Tunnel, in: Spectroscopy from Space, edited by: Demaison, J., Kluwer Academic, Dordrecht, 275-299, 2001.

Goodchild, M. F., Steyaert, L. T., Parks, B. O., Johnston, C. A., Maidment, D. R., Crane, M. P., and Glendinning, S. (Eds.): GIS and Environmental Modeling: Progress and Research Issues, Fort Collins, CO: GIS World Books, 239-242, 1996.

Janour, Z., Zelinger, Z., and Civis, S.: Laser photoacoustic spectometry and its application for simulation of air pollution in a wind tunnel, Analyst, 124, 1205-1208, 1999.

Longley, P. A., Goodchild, M. F., Maguire, D. J., and Rhind, D. W.: Geographic Information Systems and Science, John Wiley \& Sons, 27-58, New York, 2001.

Maidment, D. R., Merwade, V., Whiteaker, T., Blongevicz, M., and Arctur, D.: Time Series, in: Arc Hydro: GIS for water resources, edited by: Maidment, D. R., ESRI Press Independent Publishers Group (IPG), Redlands, California, 141-166, 2002.

Matejicek, L.: Radionuclide deposition in the Irish Sea, Proceedings of the 11th ESRI European User Conference, Imperial College, London, http://gis.esri.com/library/userconf/europroc96/ PAPERS/PN30/PN30F.HTM, 1996.

Matejicek, L.: Spatio-temporal modelling with ACSL in the GIS, Acta Universitatis Carolinae Environmentalica, 11, 55-66, 1998

Matejicek, L.: Modelling of groundwater flow networks in the desktop GIS, in: Environmental modelling the dynamics of natural, agricultural, tourism and socio-economic systems, edited by: Oxley, L. and Scrimgeour, F., Proceedings of International Congress on Modelling and Simulation Proceedings, University of Waikato, New Zealand, 55-60, 1999.

Matejicek, L.: Environmental Modelling in Urban Areas with GIS, Integrated Assessment and Decision Support, Proceedings of the 1st biennial meeting of iEMSs, Lugano, 60-65, 2002.

Rebolj, D. and Sturm, P. J.: A GIS based component-oriented integrated system for estimation, visualization and analysis of road traffic air pollution, Transport and air pollution - 8th International Symposium, 15-21, 1999.

Zelinger, Z., Strizik, M., Janour, Z., Berger, P., and Cerny, A.: Comparison of model and in-situ measurements of distribution of atmospheric pollutants, Proceedings of PHYSMOD 2003: International Workshop on Physical Modelling of Flow and Dispersion Phenomena, Italy, 6 pp., 2003. 